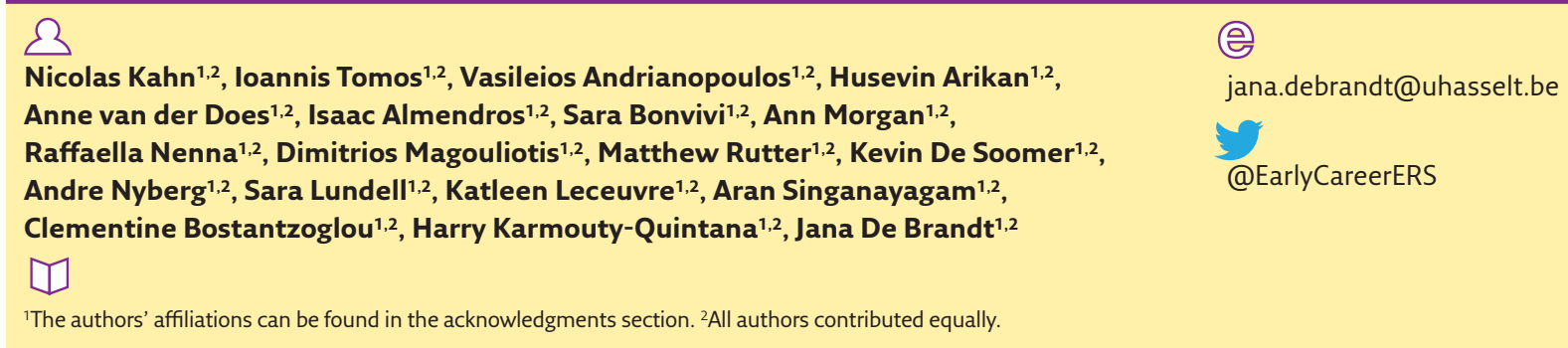

\title{
Early Career Members at the ERS International Congress 2017: highlights from the Assemblies
}

\section{Early Career Forum}

The 2017 ERS International Congress was, as always, well organised, providing participants with a good mixture of translational and clinical science. Early career members were very well represented in thematic poster, poster discussion and oral presentation sessions and were also actively involved in chairing sessions. The efforts of the Early Career Members Committee (ECMC) to increase the number of early career members included in the competence list (the list of early career members with an interest in being more actively involved in the society) paid off immensely, because the number of early career members registered improved hugely across all assemblies after the Congress. Several newly registered early career members have collated some highlights of the Congress for their assemblies, which should be of interest to all members. As assemblies 12 and 13 are new, there is no report from assembly 12 as there is not yet, at the time of writing, an early career member representative for this newly created assembly.

\section{Assembly 1}

For the first time during the ERS International Congress the ECMC, together with assemblies 1 (Clinical) and 10 (Respiratory Infections), organised a Hot Topic Session. The session was titled "Global challenges in respiratory medicine" and was designed to bring to the attention of delegates: the impact of neglected tropical diseases on lung health; the promises and challenges of new tuberculosis (TB) treatment regimens; and the different pneumococcal vaccination practices and recommendations around the world. The broad spectrum of the topics covered as well as their impact on daily clinical routine combined with excellent speakers attracted a large number of delegates. The ECMC hopes to increase its impact on the sessions and topics presented at the ERS International Congress in the future.

Other highlights of the programme from assembly 1 included a variety of sessions on
Cite as: Kahn N, Tomos I, Andrianopoulos V, et al. Early Career Members at the ERS International Congress 2017: highlights from the Assemblies. Breathe 2017; 13: e121-e129. 
interventional pulmonology. As this field progresses more and more, diverse innovative endoscopic techniques received a special focus at the Congress.

The adequacy of cytological samples of mediastinal lymph nodes obtaining using endobronchial ultrasound guided-transbronchial needle aspiration (EBUS-TBNA) for the molecular characterisation of lung adenocarcinoma was evaluated [1], and showed that EGFR mutation and ALK translocation analysis can be obtained in a high percentage of patients. In addition, in an effort to distinguish malignant from benign lymph nodes, a prospective single-centre trial evaluated the diagnostic value of endobronchial ultrasound elastography proposing it as an additional feature to the invasive EBUS-TBNA [2]

Pulmonary rehabilitation (PR) is a comprehensive intervention designed to improve physical and psychological condition, and may benefit patients with non-cystic fibrosis bronchiectasis (NCFB) [3, 4]. Evidence has shown that supervised exercise sessions (6-8 weeks) during PR improve exercise capacity, health-related quality of life and exacerbations, and decrease the need for shortacting bronchodilators in NCFB patients $[5,6]$. Nevertheless, a limited number of studies have explored the effects of PR in NCFB and, therefore, further investigation is needed. KumAR et al. [7] wanted to corroborate the efficacy of a PR programme in NCFB patients with a frequentexacerbation phenotype. They randomised 60 patients into two groups: the intervention group $(n=31)$, which was assigned an 8-week PR programme; and the control group $(n=29)$, which received clinical care without PR. Investigators assessed the changes from baseline to 8 weeks for the following outcomes: 6-min walking distance (6MWD), maximal oxygen consumption during cardiopulmonary exercise testing (CPET- $V^{\prime} \mathrm{O}_{2} \mathrm{max}$ ), spirometric indices (forced expiratory volume in $1 \mathrm{~s}$ and forced vital capacity), quality of life (St George's Respiratory Questionnaire and Leicester Cough Questionnaire (LCQ)), and the depression, anxiety and stress scale (DASS). Significantly greater improvements in the intervention group compared to the control group were detected for exercise capacity (6MWD and CPET- $V^{\prime} \mathrm{O}_{2}$ max), all dimensions of the LCQ, and the DASS scores (all $\mathrm{p} \leq 0.016$ ). Management of NCFB patients requires a comprehensive multimodal therapeutic approach [8]. According to the findings from this study, PR is highly beneficial for patients with NCFB and should be part of routine care.

\section{Assembly 2}

Assembly 2 is proud to announce that more than half the contributions to the poster sessions and oral presentations from our assembly came from our early career members. Aside from ground breaking research, one of the highlights of the Congress was the session on noninvasive ventilation (NIV) in acute respiratory failure (ARF). The new guidelines developed jointly by the ERS and American Thoracic Society (ATS) were discussed in a well-attended symposium. Stefano Nava (Bologna, Italy) and Paolo Navalesi (Milan, Italy), two of the most distinguished scientists in the field chaired the session. Bram Rochwerg (Hamilton, ON, Canada) summarised the main differences from previous guidelines. The new guideline developed recommendations for 11 questions addressing the use of NIV for various aetiologies of ARF. The answers to these questions act as a guide for clinical practice to physicians around the world. Mark Elliott (Leeds, UK) talked about recommendations and evidence for NIV in acute hypercapnic respiratory failure. In summary, the certainty of evidence is high and recommendations are strong for this indication and NIV should be considered the standard therapy in this population. Massimo Antonelli (Rome, Italy) emphasised recommendations and evidence in acute hypoxaemic respiratory failure. There is strong evidence suggesting a benefit of NIV for this patient group, but specific details need to be further addressed in future studies. The symposium finished with a presentation by Nicholas Hill (Boston, MA, USA), who provided information regarding technical issues around NIV. Both the speakers and chairs of the session indicated that the evidence around NIV is evolving so rapidly that, with new information coming from ongoing studies, the recommendations might already change in the near future. Furthermore, new emerging therapies such as high-flow oxygen systems and extracorporeal carbon dioxide removal may replace NIV for some indications.

\section{Assembly 3}

One of the highlights of assembly 3 at the 2017 ERS International Congress in Milan was the talk given by Renat Shaykhiev (New York, NY, USA) during the highly interesting session on lung tissue repair and remodelling in chronic lung diseases, in the basic translational science track.

Renat Shaykhiev's talk addressed very fundamental and important questions regarding the role of basal cells in the aberrant repair of the epithelium in chronic obstructive pulmonary disease (COPD). By performing transcriptome profiling of bronchoscopic brushes, region-specific gene signatures of the airways were identified that were subsequently found to be different in patients with COPD when compared to controls. The data discussed showed how the epithelial composition of the distal airways of patients with COPD showed enhanced levels of markers for proximal airways (e.g. increased stratification and increased numbers of mucus producing cells), thereby mimicking the proximal airway epithelium of the airways of nonsmokers [9]. Furthermore, they detected a significant expansion of the vascular niche in COPD, especially 
around basal cells. Single-cell RNA-sequence analysis revealed three main clusters: an epithelial, stromal and immune cell cluster. The epithelial cell cluster showed expression of a unique set of factors specific for this pre-terminal part of the human lung. These data allowed transcriptome "lineage tracing", identifying one overall marker for airway epithelial cells, KRT19, and further identifying markers for several intermediate cell states reflecting distinct stages of epithelial differentiation.

Renat Shaykhiev's group is also interested in the cross-talk between stromal cells and airway epithelial cells. Co-cultures of primary epithelial cells cultured at the air-liquid interface in the presence of (healthy) stromal cells show enhanced levels of ciliated and secretory epithelial cells, compared to cultures of airway epithelial cell alone. When performing these cultures with cells derived from COPD patients, the epithelial cells changed to a more proximal airway-like phenotype, which was also confirmed in organoids. Lastly, they found a significant loss of physiological communication between airway epithelial cells and stromal cells in COPD, as was shown by performing mixed co-cultures (e.g. healthy-derived airway epithelial cells and COPD-derived stromal cells and vice versa). Interestingly, healthy stromal cells could restore aberrant regeneration of the airway epithelium derived from COPD patients.

In conclusion, these data suggest that epithelial dysfunction in COPD might not be associated with a molecular defect in epithelial cells alone, but is also dependent on interactions with airway stromal and other cells present in the microenvironment.

\section{Assembly 4}

The 2017 ERS International Congress held in Milan provided an excellent opportunity to discover the novel insights obtained from basic, translational and clinical studies on respiratory physiology and sleep apnoea. As usual, the different scientific sessions pointed out the importance of a multidisciplinary approach to the assessment of patients with sleep disordered breathing and to understand respiratory physiology. In this regard, the usefulness and optimisation of automated positive airway pressure, mandibular advancement devices and surgical treatment for sleep apnoea patients were discussed in the symposium "Multidisciplinary management of sleep apnoea". This symposium was also complemented by a postgraduate course on the diagnosis and treatment of obstructive sleep apnoea (OSA) and a meet the expert session focused on OSA syndrome in children.

Regarding sleep quality and habits, it is important to mention the hot topic session organised to discuss the implications of the circadian molecular clock in the pathogenesis and treatments of different respiratory diseases. This is a promising field of study, which needs more attention to better understand how sleep disruption can alter the biological clock. Jeffrey C. Hall, Michael Rosbash and Michael W. Young have been recognised this year with the 2017 Nobel Prize in Physiology or Medicine for their work discovering of the molecular mechanisms controlling the circadian rhythm highlighting the importance of this field.

"The best of the ERS/ESRS Congress 2017" was a very attractive session to assembly 4 members. This session addressed different topics including the use of neuroimaging to assess brain structure and activation in patients treated for OSA, insomnia in sleep disordered breathing, and the coexistence of OSA with other respiratory and cardiovascular diseases. In accordance with this session, a large number of mechanistic studies were presented that provided new insights into the cardiovascular, neurological and metabolic consequences of OSA during thematic poster sessions, which led to animated discussions. In the oral presentation session "New insights into the understanding of sleep and the consequences of sleep apnoea" very interesting and diverse works related to cancer, cardiac ischaemia, genome methylation and the release of cell-free DNA and nucleosomes in OSA patients were presented, among other innovative works.

This year, a meet the expert session was organised focusing on high altitude medicine. The studies presented in this session provided new information to the audience to better understand the immediate adjustments and acclimatisation mechanisms to high altitude. In this field, S. Vergès (Grenoble, France) and co-workers presented the MEDEX 2015 study showing that positive expiratory pressure can improve oxygenation and symptoms at high altitude. Other important work based on respiratory and exercise physiology was also presented in different sessions. In this regard, Rossiter et al. [10] have shown that genetic variants predicting the response to endurance exercise training are associated with skeletal muscle oxidative capacity in COPD. Meanwhile, a study carried out by CHLUMSKY et al. [11] presented the limitations of exercise tolerance in patients with idiopathic pulmonary fibrosis.

\section{Assembly 5}

Airway sensory nerves drive a number of reflexes in the airway such as cough and bronchoconstriction, which are key symptoms of asthma and COPD. Cough, in particular, currently has no safe and effective therapies and novel treatments are urgently required. The ERS International Congress in Milan showcased the role of the purinergic $\mathrm{P} 2 \mathrm{X} 2 / 3$ receptor in a number of clinical and preclinical studies. The P2X3 antagonist MK7264 was trialled in 253 chronic cough patients in a double centre clinical trial. In the study by SMITH et al. [12] 63 patients received placebo, 64 patients received $7.5 \mathrm{mg}$ MK7264, 63 patients received $20 \mathrm{mg}$ and 
63 patients received $50 \mathrm{mg}$. MK7624 was shown to significantly inhibit $24 \mathrm{~h}$ cough frequency in a concentration-dependant manner, and at concentrations which limited the most common side-effect of dysgeusia, highlighting this receptor as a good therapeutic target. Preclinical work, by Bonvivı [13] et al., demonstrated that a suitable challenge agent to test this pathway is a hypoosmolar solution, as sensory nerve depolarisation induced by solutions of lower osmolarity was inhibited by a P2X3 antagonist. Indeed, MORICE et al. [14] also showcased that MK7264 was able to inhibit cough in hypo-osmolar and ATP-challenged patients, indicating that this challenge agent could be used to characterise patient types who may respond well to $\mathrm{P} 2 \mathrm{X} 3$ treatment.

Another key topic of this Congress was the use of e-cigarettes or vaping. In one study by SHARMA et al. [15], mice were exposed to e-cigarette vapour and it was indicated that the offspring of these mice were at more risk of developing allergic asthma. Tobacco smoke is already known to increase asthma risk, but for the first time this study demonstrated that e-cigarette vapour can lead to impaired lung function and increased risk of asthma in the offspring, highlighting potential risks of e-cigarette usage [15]. However, nicotinecontaining e-cigarettes have also been shown to be antitussive, and a further interesting study indicated that this antitussive effect is mediated through the alpha7 nicotinic receptor, as agonists of this receptor had the same effect [16]. This highlights another non-opioid therapeutic option for the treatment of cough.

\section{Assembly 6}

This year's ERS Congress provided a wealth of sessions of interest to members of Assembly 6 (Epidemiology and environment), especially pulmonologists, clinicians and other health professionals faced with treating the complex cardiopulmonary patient. Cardiovascular disease comorbidities in people with COPD provided the focus for an interesting and well-attended symposium which reviewed the epidemiological evidence for the association between the two diseases as well as that for the efficacy of the various treatment options, including smoking cessation. L. Fabbri (Modena, Italy), drew attention to the scale of underdiagnosis of coexisting cardiovascular diseases in people with COPD - and vice versa using observational data on heart failure to illustrate his point. According to a recent study by F. Franssen (Horn, the Netherlands) and co-workers, more than $70 \%$ of patients attending cardiovascular outpatient clinics who were subsequently found to have airflow limitation had not previously received spirometry [17]. L. Fabbri also drew some interesting parallels between the treatment algorithms for heart failure and COPD which served to highlight the key message of his talk, namely that COPD is the lung component of multimorbidity and it is vital that we look beyond the lungs and treat comorbidities if we wish to improve the quality of life for people living with COPD [18].

Another focus was the need for biomarkers in order to stratify COPD patients according to their cardiovascular disease risks as a means to guide therapy. In this respect, it seems that people with COPD are at a distinct disadvantage compared with those with asthma. At the moment there is no biomarker suitable for use in COPD, and although it might be possible to derive better prognostic capabilities by using a combination of biomarkers, the speaker expressed his view that there is a long way to go before this sort of approach could be adopted in clinical practice.

Other interesting presentations focused on the treatment of COPD. In the absence of new drugs for COPD, considerable attention is given to alternative management strategies, including smoking cessation, PR and physical activity. These issues were covered in a number of different sessions at this year's Congress, all of which highlighted the fact that while there is good evidence of benefit, uptake remains poor.

\section{Assembly 7}

The presentations on paediatric respiratory diseases were of very high quality this year for both the educational and the scientific sessions. In the postgraduate course on paediatric asthma, participants were guided in the approach to the pathology from allergic sensitisation and wheezing. Numerous sessions in collaboration with adult pulmonologists focused on the issue of severe asthma management and NCFB. A particularly impressive session was "Omics in paediatric asthma and beyond". In this session presenters collected the available data on genetics and epigenetics, metabolomics and microbiome factors in asthma. In particular, several recent genome-wide association studies reported information about the various new pathways that may be implicated in asthma pathophysiology. By contrast, the metabolic profile of a patient with asthma has been demonstrated to differ from healthy patients and to vary according to disease severity. Finally, microbes have been shown to play a role in asthma development and in the heterogeneity of the disease. In the hot topics session "Early life events: implications across the lifecourse", the role of early life determinant factors such as the microbiome, infections and bronchopulmonary dysplasia on respiratory health in later life were elucidated. At the meet the expert session focused on OSA syndrome in children, updates for physicians treating children with OSA syndrome were comprehensively addressed. Finally, the high educational level of clinical cases presented during the paediatric grand round allowed all young 
and senior participants to try to diagnose and approach the management of cases of particular interest in the paediatric respiratory field.

In the "Paediatric year in review" session the updated scientific literature about four topics was presented: chronic interstitial lung disease in children, viral infections in wheezing disorders, pulmonary vascular development in relation to congenital lung disease and the ERS statement on interventional bronchoscopy.

\section{Assembly 8}

The 2017 ERS International Congress in Milan provided presentations and abstracts of high quality in the field of thoracic surgery and lung transplantation. One of the most interesting poster discussion sessions for assembly 8 was "From donor lung selection and organ preservation to lung transplant outcome", which included a wide variety of posters that raised debate. The poster "Evaluation of a lung donor score: retrospective analysis of Swiss donor data" [19] assessed the lung donor quality score as described by SMITS et al. [20] in order to extend the lung donor pool. The authors suggested that their adapted lung donor score reflects to a certain extent the suitability for transplantation of the donor lung. Another topic of numerous posters was extracorporeal membrane oxygenation. One poster, "Intraoperative extracorporeal membrane oxygenation versus cardiopulmonary bypass for lung transplantation: a meta-analysis" [21], compared these two methods of intraoperative cardiopulmonary support during lung transplantation. The authors suggested that intraoperative extracorporeal membrane oxygenation for lung transplantation was associated with better clinical outcomes in terms of intubation time $(p<0.00001)$, intensive care unit stay $(p<0.0001)$, hospital stay $(p<0.00001)$ and primary graft dysfunction $(p<0.003)$. There was no difference in mortality at 1,3 and 12 months $(\mathrm{p}=0.72,0.41$ and 0.62 , respectively).

Two interesting posters looked at the role of computed tomography (CT) after lung transplantation. SALITO et al. [22] supported the reliability of specific gas volume analysis as an attractive quantitative $\mathrm{CT}$ parameter of lung function after lung transplantation, while in a second poster the same group proposed that $\mathrm{CT}$ regional analysis may provide a significant advance in the interpretation of ventilation abnormalities after lung transplantation [23]. Another topic that was assessed by a team from Leuven, Belgium was the impact of genetic variations and polymorphisms on the outcome after lung transplantation. HEIGL et al. [24] presented findings regarding the association of STAT3 genetic variations with patient survival probability after lung transplantation. In the same context, BeLLon et al. [25] showed that underlying polymorphisms of MMF transporters affected daily immunosuppression and were associated with primary outcome of lung transplantation. This result highlights the importance of dosage and monitoring of immunosuppressive drugs.

\section{Assembly 9}

The symposium, organised by the European Lung Foundation (ELF), on "Air travel for respiratory patients", which revealed the challenges patients, clinicians and air travel providers deal with when patients travel and need to organise additional oxygen, was very well received within the lung function technologist group (9.1). Matthew Rutter, a respiratory physiologist and himself a patient, testified about the overlooked difficulties faced by individuals with dyspnoea and hypoxia when travelling to the Congress. Another symposium provided the latest insights on the GLI (Global Lung Function Initiative) reference values. The session opened with an overview of the life of Prof. Philip Quanjer, founder of the Global Lung Function Initiative, who sadly passed away this summer. The importance of the GLI reference values for spirometry was emphasised. It was possible to gain a much better understanding of the difficulties in producing reference values and to obtain an update to the considerable volume of work that has been, and is continuing to be, undertaken. New reference values on diffusion capacity were presented [26] and the present mission of the GLI committee to collect data on static lung volumes was introduced.

Other interesting topics were a poster session discussing lung function testing in different patient categories, including obese patients, COPD patients and even members of the European Commission, and an interesting presentation during the session on "Technique and technology in lung function" revealing that it is achievable to assess intrinsic positive end-expiratory pressure with body plethysmography [27]. Animated and interesting discussions were ignited during a presentation on artificial intelligence applied to lung function outcomes as a tool to improve diagnostic accuracy in the "Best abstracts in lung function" session, and in the thematic poster sessions, where one suggestion was quite controversially: "spirometry may be soon superseded by other tests that require less patient effort and give more information, such as impulse oscillometry/forced oscillation technique and resistance volume loops". However, while spirometry may not be replaced it will be complimented. The manufacturers in the exhibition were demonstrating their latest updates and even provided educational sessions, which were found to be invaluable in allowing clinicians to implement changes to practice immediately.

From the perspective of the physiotherapy group (9.2), Congress highlights included a well-attended postgraduate course that provided the latest insights in novel interventional tools and rehabilitation 
strategies for locomotor and respiratory muscles. The latter was a recurrent topic at this year's Congress. Specifically, the effects of inspiratory muscle training were evaluated across several respiratory diseases and the association between respiratory function and core muscle strength was discussed. Furthermore, the importance of physical activity among patients with respiratory diseases was emphasised. For example, among patients with COPD a higher physical activity level was associated with lower mortality risks across the Global Initiative for Chronic Obstructive Lung Disease stages. On the topic of physical activity, the effects of novel intervention strategies, including but not limited to urban training strategies as well as eHealth interventions, were investigated across several studies. Furthermore, the use of (and effects on) functional outcomes as well as the importance of quality (not only quantity) of exercise training to optimise effects were other areas highlighted across symposiums, oral and poster sessions during the Congress. Finally, the ELF/ERS award for best abstract in patient-centred research was received in the physiotherapy group for an abstract about COPD care in primary care [28]

The nursing group (9.3) organised many very interesting topics and speakers. It was impossible to see them all, but one thing was very striking: all topics were well organised and professionally presented. Evidence based, convincing new ideas where brought forward by motivated nurses and other health-care professionals. Katleen Leceuvre (Leuven, Belgium), an early career member from the nursing group, proudly accepted the challenging task of chairing a poster discussion, which gave impressive and new insights on very different topics: end-of-life care, maintaining quality of life, different and new forms of follow-up of patients, bronchoscopy and many other topics. All were presented by enthusiastic nurses from all over the world. The input of nurses at the ERS International Congress is invaluable. It encourages a critical mind, enhances problem-solving and improves clinical reasoning. Knowledge leads to professional development. Equally important are the interactions and conversations with other nurses and healthcare professionals. New perspectives and collaboration can create new pathways of care for the respiratory patient. Still, there is some work to be done. The importance of specialised nurses as a part of the multidisciplinary team deserves more attention. Most important however is the patient, who has to be at the centre of our attention, guidance and professional care.

\section{Assembly 10}

The increasingly important field of the lung microbiome and its role in health and disease featured prominently at this year's Congress with a prestigious joint ERS/ATS session dedicated to this subject. Notable presentations focused on hostmicrobiome interactions in respiratory health and in the context of chronic respiratory diseases such as COPD.

A pro-con debate session on current controversies in community-acquired pneumonia stimulated much interest with hot topics including the role of steroids in community-acquired pneumonia management, antibiotic strategies including use of macrolides and the role of thoracic ultrasound in diagnosis discussed and debated at length.

TB also featured prominently and there was a focus on the increasing global burden of the disease with presentations dedicated to novel anti-tuberculous therapies for multidrugresistant disease currently in development. These novel therapeutic options provide hope that the multidrug-resistant TB epidemic can be curbed in the future. Non-tuberculous mycobacterial disease remains a therapeutic challenge clinically and a symposium on this topic provided useful insight on the natural history of the disease and complexities related to diagnosis and management.

Interest in the area of NCFB continues to grow and various updates were provided from the EMBARC (European Bronchiectasis Registry) study group, an ERS collaborative registry which has recruited a large database of patients across Europe. The group provided novel insight into the set-up, collaborations and external funding acquired to date as well as providing novel data on the association between gastro-oesophageal reflux and exacerbations of bronchiectasis.

\section{Assembly 11}

The 2017 ERS International Congress featured exciting updates and educational sessions in Thoracic oncology.

In a well-attended state of the art symposium on lung cancer, the head of assembly 11, Nir Peled (Hod Hasharon, Israel), presented the current treatment approach for nonsmall cell lung cancer. Accurate diagnosis remains crucial as we move towards tailored treatment modalities. Development of effective screening programmes, circulating tumorous DNA technology, implementation of genetics and proteomics technology to predict response to therapy, integration of novel immunotherapeutic agents in every day practice, and developing a better understanding of resistance mechanisms are key factors in the era of precision medicine for lung cancer patients.

During the Chinese Programme session, ERS President, Mina Gaga (Athens, Greece) provided insights on current knowledge on lung cancer screening programmes. The National Lung Screening Trial (NLST), the largest lung cancer screening study published to date, showed that $20 \%$ more patients were diagnosed with low-dose CT and 
$50 \%$ more patients were diagnosed at stage I-II, where treatment is with curative intent. However, there are important issues to be addressed such as selection of individuals for screening, false positive results, over-diagnosis and management of positive findings.

A postgraduate course was dedicated to the leading role of chest physicians in diagnostics and therapy of lung cancer. Matthew Evison (Manchester, UK) discussed the current strategies followed to assess fitness for radical treatment in lung cancer. National and international guidelines were reviewed, followed by casebased discussion. Jan van Meerbeeck (Antwerp, Belgium) presented the new staging system and its implications for multimodal treatment options. Changes in the new edition of the staging system were thoroughly analysed through interactive discussion of challenging cases. David Baldwin (Nottingham, UK) reviewed principles and evidence of CT screening and noninvasive diagnostics. Based on the promising results of NLST trial in reduction of the lung cancer mortality rate, despite the considerable issues in study design, Prof. Baldwin presented numerous policies that need to be implemented for more effective screening programmes, including more elaborate selection criteria, radiological techniques such as automated volumetry, optimal multidisciplinary work-up for screening programme participants and integration of smoking cessation programmes.

In an oral presentation session, Marc van der Schee, on behalf of the LuCID consortium, presented the LuCID study, which evaluates the use of breath biomarker analysis for early detection of lung cancer [29]. This international multicentre prospective case-control cohort study, has recruited, to date, over 1500 patients with clinical suspicion of lung cancer. Volatile organic compounds (VOCs) present in patients' exhaled breath are collected and analysed, using the Breath Biopsy platform (Owlstone Medical, Cambridge, UK), to determine whether VOC biomarkers in breath can be used to discriminate between patients with and without lung cancer. LuCID aims to pave the way for the development of a noninvasive breath test that could improve screening and early detection rates.

Immunotherapy for lung cancer was discussed in multiple sessions. During a symposium, Joachim G.J.V. Aerts (Rotterdam, Netherlands) underlined the need to incorporate preclinical science into clinical studies, in order to deliver personalised immune-oncological therapies. Joanna DomagalaKulawik (Warszawa, Poland) presented recent data on immune responses in oncology, and also stressed the need for research towards discovering markers to schedule treatment, anticipate response, overcome escape mechanisms, evaluate tumour progression and even predict immune-related adverse events.

A postgraduate course was dedicated to pleural disease management. Julius Janssen (Nijmegen, the
Netherlands) reviewed the current diagnostic and treatment approach for malignant pleural effusion (MPE) and Nick Maskell (Bristol, UK) presented modern strategies for pneumothorax management. During a symposium dedicated to reviewing recent randomised controlled trials in management of MPE, Rahul Bhatnagar (Bristol, UK) presented results from the IPC-PLUS trial which examined the efficacy of indwelling pleural catheter (IPC) placement versus IPC placement plus sclerosant (talc) in patients with MPE managed as outpatients. The trial results support the administration of talc via an IPC, on an outpatient basis, as a safe method that doubles success rates for pleurodesis. An interesting oral presentation was given by loannis Psallidas (Oxford, UK) on a new prognostic score to predict 3-month mortality for patients with MPE [30]. The score, named PROMISE, comprises of haemoglobin, C-reactive protein, white blood cell count, Eastern Cooperative Oncology Group performance status, type of cancer, TIMP-1 concentration in pleural fluid, and history of chemotherapy and radiotherapy. It was found to perform well in both internal and external validation.

\section{Assembly 13}

At the ERS International Congress in Milan, a new assembly dedicated to pulmonary vascular diseases was introduced to the ERS structure. The Congress already covered exciting research in this field, for example a very interesting oral presentation session on "New perspectives from experimental models of pulmonary hypertension".

Advances in basic research provided further insights into the role of the BMPR2 signalling in the development of pulmonary hypertension $(\mathrm{PH})$. In a study presented by Ayumi Sekine, pharmacological or genetic deletion of BMP9, a ligand of BMPR2 and the ALK1 receptor complex, attenuated the development of $\mathrm{PH}$ in two distinct experimental models [31]. Also in BMPR2 biology, Alexi Crosby demonstrated a novel two-hit model of $\mathrm{PH}$ where $\mathrm{BMPR} 2^{+/-}$mice received multiple doses of lipopolysaccharide leading to increased right ventricle systolic pressure (RVSP) [32]. Using this novel inflammatory model, the study demonstrated that the bone marrow cells from $\mathrm{BMPR}^{+/-}$mice were pivotal in the development of $\mathrm{PH}$. The intriguing mechanism proposes that BMPR2 defects in bone marrow cells lead to increased megakaryocytes and platelets that release proangiogenic and vasoconstrictory mediators. Taken together these studies uncover novel mechanisms of BMPR2-modulated PH and provide further rationale to study the role of BMPR2 in inflammatory cells.

A promising study presented by Kathryn Wilson demonstrated that an apoptosis signal-regulating kinase 1 (ASK1) inhibitor was able to reverse RVSP and vascular remodelling in a rat model 
of sugen-hypoxia induced $\mathrm{PH}$ [33]. This study provided a direct translational link to a session highlighting new clinical trials results in $\mathrm{PH}$ therapy. In this session the results from the ARROW study that evaluated the effect of selonsertib, an ASK1 inhibitor, on group $1 \mathrm{PH}$ were presented [34]. Despite the encouraging preclinical results, the main conclusion of the ARROW study was that although well tolerated selonsertib did not appear to provide any clinical benefit to patients with $\mathrm{PH}$. Also in this session, results from the RESPITE study presented by Marius Hoeper showed that riociguat, a drug approved to treat group 1 and group $4 \mathrm{PH}$, was also effective at targeting patients resistant to PDE5i-therapy [35]. Intriguingly, in the RISEIIP study presented by Steven Nathan, where the efficacy of riociguat was evaluated in patients with $\mathrm{PH}$ and interstitial lung disease (classified as group $3 \mathrm{PH}$ ) the trial was terminated early due to clinical worsening in patients that received riociguat [36]. The differences in the efficacy of riociguat in the RESPITE and RISE-IIP studies highlights the mechanistic differences between group 1 and group $3 \mathrm{PH}$ and underscores the need to develop further research efforts in understanding the pathophysiology in group $3 \mathrm{PH}$ where no approved therapies exist.

In conclusion, this session elegantly showed the latest research and very encouraging results in different aspects of $\mathrm{PH}$ research.

\section{Concluding remarks}

Overall, this year's ERS International Congress has proven to be the place where science, clinic education and lifestyle meet. The Congress provided many opportunities for early career members to develop their career and to get involved in the society. We would like to invite those early career members who have never attended the ERS International Congress before to join us in Paris next year. We encourage all early career members that would like to be involved in the society to register on the competence list by contacting their ECMC representative.

\section{Acknowledgements}

The authors' affiliations are as follows. Nicolas Kahn: Dept of Pneumology and Critical Care Medicine, Thorax-klinik, University Hospital Heidelberg, Heidelberg, Germany; Ioannis Tomos: 2nd Pulmonary Medicine Dept, "ATTIKON” University Hospital, National and Kapodistrian University of Athens, Athens, Greece; Vasileios Andrianopoulos: Dept of Respiratory Medicine and Pulmonary Rehabilitation, Schoen Klinik Berchtesgadener Land, Schoenau am Koenigssee, Germany; Husevin Arikan: Marmara University Hospital, Respiratory and Critical Care Medicine, Istanbul, Turkey; Anne van der Does: Dept of Pulmonology, Leiden University Medical Center, Leiden, The Netherlands; Isaac Almendros: Unitat de Biofísica i Bioenginyeria, Facultat de Medicina i Ciències de la Salut, Universitat de Barcelona, Barcelona, Spain, Centro de Investigación Biomédica en Red de Enfermedades Respiratorias, Madrid, Spain and Institut d'Investigacions Biomèdiques August Pi i Sunyer (IDIBAPS), Barcelona, Spain; Sara Bonvivi: Respiratory Pharmacology Group, Airway Disease Section, National Heart and Lung Institute, Imperial College London, London, UK; Ann Morgan: Respiratory Epidemiology, Occupational Medicine and Public Health, National Heart and Lung Institute, Imperial College London, London, UK; Raffaella Nenna: Dept of Pediatrics and Infantile Neuropsychiatry, "Sapienza" University of Rome, Rome, Italy; Dimitrios Magouliotis: Dept of Surgery, University Hospital of Larissa, Larissa, Greece; Matthew Rutter: Lung Function Dept, Cambridge University Hospitals NHS Foundation Trust, Cambridge, UK; Kevin De Soomer: Dienst Longziekten, University Hospital Antwerpen, Edegem, Belgium; Andre Nyberg: Dept of Community Medicine and Rehabilitation, Physiotherapy, Umeå University, Umeå, Sweden and Institut universitaire de cardiologie et de pneumologie de Québec, Québec, QC, Canada; Sara Lundell: Dept of Community Medicine and Rehabilitation, Physiotherapy, Umeå University, Umeå, Sweden; Katleen Leceuvre: Pneumology, University Hospital Leuven, Leuven, Belgium; Aran Singanayagam: Airways disease infection, National Heart and Lung Institute, Imperial College London, London, UK; Clementine Bostantzoglou: 7th Respiratory Medicine Dept, "Sotira" Athens Chest Hospital, Athens, Greece; Harry Karmouty-Quintana: Dept of Biochemistry and Molecular Biology, UTHealth - McGovern Medical School, Houston, TX, USA; Jana De Brandt: REVAL Rehabilitation Research Center, BIOMED - Biomedical Research Institute, Faculty of Medicine and Life Sciences, Hasselt University, Diepenbeek, Belgium

\section{Support statement}

Vasileios Andrianopoulos is the recipient of an ERS-EU RESPIRE2 Marie Sklodowska-Curie Postdoctoral Research Fellowship-Number MCF (8465)-2015.

\section{Conflict of interest}

Disclosures can be found alongside this article at breathe.ersjournals.com 


\section{References}

1. Fuso L, Magnini D, Varone F, et al. Adequacy of EBUS-TBNA specimens for molecular testing in lung adenocarcinoma. Eur RespirJ 2017; 50: Suppl. 61, PA837.

2. Gompelmann D, Kontogianni K, Sarmand N, et al. Elastography in the diagnosis of hilar and mediastinal lymph nodes. Eur Respir J 2017; 50: Suppl. 61, PA828.

3. Spruit MA. Pulmonary rehabilitation. Eur Respir Rev 2014; 23 : 55-63.

4. Zanini A, Aiello M, Adamo D, et al. Effects of pulmonary rehabilitation in patients with non-cystic fibrosis bronchiectasis: a retrospective analysis of clinical and functional predictors of efficacy. Respiration 2015; 89: 525-533.

5. Ong HK, Lee AL, Hill CJ, et al. Effects of pulmonary rehabilitation in bronchiectasis: a retrospective study. Chron Respir Dis 2011; 8: 21-30.

6. Al Moamary MS. Impact of a pulmonary rehabilitation programme on respiratory parameters and health care utilization in patients with chronic lung diseases other than COPD. East Mediterr Health J 2012; 18: 120-126.

7. Kumar R, Guleria R, Khilnani GC, et al. The effects of pulmonary rehabilitation in patients with non-cystic fibrosis bronchiectasis - a randomised controlled trial. Eur Respir J 2017; 50: Suppl. 61, OA307.

8. McShane PJ, Naureckas ET, Tino G, et al. Non-cystic fibrosis bronchiectasis. Am J Respir Crit Care Med 2013; 188: 647-656.

9. Yang J, Zuo W-L, Fukui T, et al. Smoking-dependent distalto-proximal repatterning of the adult human small airway epithelium. Am J Respir Crit Care Med 2017; 196: 340-352.

10. Rossiter H, Adami A, Cho M, et al. Genetic variants predicting the response to endurance exercise training are also associated with skeletal muscle oxidative capacity in COPD. Eur RespirJ 2017; 50: Suppl. 61, OA3428.

11. Chlumsky J, Vasakova M, Sterclova M, et al. Limitations of exercise tolerance in patients with IPF. Eur Respir J 2017; 50: Suppl. 61, OA1965

12. Smith J, Kitt $M$, Morice $A$, et al. Inhibition of $P 2 \times 3$ by MK-7264 reduces 24-hour cough frequency in a randomized, controlled, Phase 2b clinical trial. Eur RespirJ 2017; 50: Suppl. 61, OA2932.

13. Bonvini S, Dubuis E, Adcock J, et al. Activation of transient receptor potential (TRP) channels by hypoosmolar solution: an endogenous mechanism of ATP release and afferent nerve activation. Eur Respir J 2017; 50: Suppl. 61, OA4410.

14. Morice $A H$, Kitt M, Ford A, et al. The Effect of MK-7264, a $\mathrm{P} 2 \mathrm{X} 3$ antagonist on cough reflex sensitivity in a randomized crossover trial of healthy and chronic cough subjects. Eur Respir J 2017; 50: Suppl. 61, OA2931.

15. Sharma $\mathrm{P}$, McAlinden $\mathrm{K}$, Chan $\mathrm{Y}$, et al. Maternal eCigarette vaping enhances Th2 driven asthma in the offspring. Eur Respir J 2017; 50: Suppl. 61, PA4694

16. Dicpinigaitis $\mathrm{P}$, Canning $\mathrm{B}$, DeVita $\mathrm{R}$, et al. The antitussive effects of alpha7 $(\alpha 7)$ nicotinic receptor agonists. Eur Respir J 2017; 50: Suppl. 61, OA4409.

17. Franssen FM, Soriano JB, Roche N, et al. Lung function abnormalities in smokers with ischemic heart disease. Am J Respir Crit Care Med 2016; 194: 568-576.

18. Fabbri LM. Smoking, not COPD, as the disease. $N$ Engl J Med 2016; 374: 1885-1886.

19. Elmer A, Weiss J, Aubert J-D, et al. Evaluation of a lung donor score: retrospective analysis of Swiss donor data. Eur RespirJ 2017; 50: Suppl. 61, PA1543.
20. Smits JM, van der Bij W, Van Raemdonck D, et al. Defining an extended criteria donor lung: an empirical approach based on the Eurotransplant experience. Transpl Int 2011; 24: 393-400

21. Magouliotis D, Svokos A, Svokos K, et al. Intraoperative extracorporeal membrane oxygenation versus cardiopulmonary bypass for lung transplantation: a meta-analysis. Eur Respir J 2017; 50: Suppl. 61, PA1550.

22. Salito C, Pennati F, Aliverti A, et al. Reliability of computed tomography (CT) quantitative analysis in lung transplantation follow-up. Eur Respir J 2017; 50: Suppl. 61, PA1556.

23. Salito C, Pennati F, Palleschi A, et al. Regional analysis with quantitative computed tomography after lung transplantation. Eur Respir J 2017; 50: Suppl. 61, PA1557.

24. Heigl T, Bellon $H$, Sacreas $A$, et al. Genetic variation in the STAT3 gene has an impact on survival after lung transplantation. Eur Respir J 2017; 50: Suppl. 61, PA1559.

25. Bellon $H$, Vandermeulen $E$, Van Herck A, et al. Polymorphisms in immunosuppression transporters affect outcome after lung transplantation. Eur Respir J 2017; 50: Suppl. 61, PA1560.

26. Stanojevic S, Graham BL, Cooper BG, et al. Official ERS technical standards: Global Lung Function Initiative reference values for the carbon monoxide transfer factor for Caucasians. Eur Respir J 2017; 50: 1700010.

27. Radovanovic D, Zilianti C, Santus P, et al. Plethysmographic measurement of intrinsic PEEP in stable COPD patients at rest. Eur RespirJ 2017; 50: Suppl. 61, PA3014.

28. Lundell S, Tistad M, Rehn B, et al. Mapping COPD care in primary care - professionals' perspective. A mixed methods study. Eur RespirJ 2017; 50: Suppl. 61, PA2526.

29. van der Schee MPC, Boschmans J, Smith R, et al. Early detection of lung cancer through analysis of VOC biomarkers in exhaled breath: the LuCID study. Eur RespirJ 2017; 50: Suppl. 61, OA1472.

30. Psallidas I, Kanellakis N, Thezenas $M$, et al. Late Breaking Abstract - Prognostic and therapeutic markers of malignant pleural effusion - The PROMISE study. Eur Respir J 2017; 50: Suppl. 61, OA292.

31. Sekine A, Tu L, Mallet C, et al. Contribution of BMP9 to pulmonary arterial hypertension. Eur Respir J 2017; 50: Suppl. 61, OA4658.

32. Crosby A, Toshner $\mathrm{M}$, Soon $\mathrm{E}$, et al. Bone marrow transplantation reduces susceptibility to pulmonary hypertension in BMPR2 deficient mice. Eur Respir J 2017; 50: Suppl. 61, OA4661.

33. Wilson $\mathrm{K}$, Buist $\mathrm{H}$, Jayasekera $\mathrm{G}$, et al. Reversal of pulmonary hypertension in a sugen/hypoxic rat model using an ASK1 inhibitor. Eur Respir J 2017; 50: Suppl. 61, OA4663.

34. Rosenkranz S, Feldman J, McLaughlin V, et al. The ARROW study: a phase 2 , prospective, randomized, doubleblind, placebo-controlled study of Selonsertib in subjects with pulmonary arterial hypertension. Eur Respir J 2017; 50: Suppl. 61, OA1983.

35. Hoeper MM, Corris PA, Ghofrani H-A, et al. Effect of riociguat on pulmonary arterial compliance in patients with pulmonary arterial hypertension (PAH) in the RESPITE study. Eur Respir J 2017; 50: Suppl. 61, OA1980.

36. Nathan S, Behr J, Collard HR, et al. RISE-IIP: Riociguat for the treatment of pulmonary hypertension associated with idiopathic interstitial pneumonia. Eur Respir J 2017; 50: Suppl. 61, OA1985. 